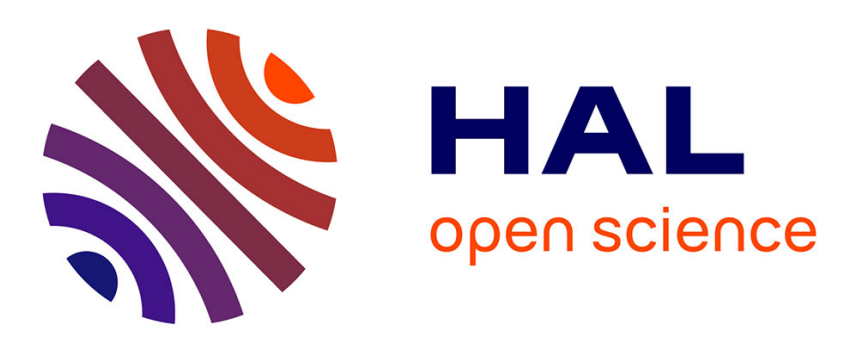

\title{
High-field mid-infrared pulses derived from frequency domain optical parametric amplification
}

\author{
Adrien Leblanc, Gilles Dalla-Barba, Philippe Lassonde, Antoine Laramée, \\ Bruno E Schmidt, Eric Cormier, Heide Ibrahim, F. Légaré
}

\section{To cite this version:}

Adrien Leblanc, Gilles Dalla-Barba, Philippe Lassonde, Antoine Laramée, Bruno E Schmidt, et al.. High-field mid-infrared pulses derived from frequency domain optical parametric amplification. Optics Letters, 2020, 45 (8), pp.2267. 10.1364/OL.389804 . hal-02877690v2

\section{HAL Id: hal-02877690 \\ https://hal.science/hal-02877690v2}

Submitted on 20 Nov 2020

HAL is a multi-disciplinary open access archive for the deposit and dissemination of scientific research documents, whether they are published or not. The documents may come from teaching and research institutions in France or abroad, or from public or private research centers.
L'archive ouverte pluridisciplinaire HAL, est destinée au dépôt et à la diffusion de documents scientifiques de niveau recherche, publiés ou non, émanant des établissements d'enseignement et de recherche français ou étrangers, des laboratoires publics ou privés. 


\title{
High-field mid-infrared pulses derived from frequency domain optical parametric amplification
}

\author{
A. Leblanc, ${ }^{1,2}$ G. Dalla-Barba ${ }^{3}$ P. Lassonde, ${ }^{1}$ A. Laramée, ${ }^{1}$ B. E. Schmidt, ${ }^{1}$ \\ E. Cormier, ${ }^{3,4}$ H. IBrahim, ${ }^{1}$ (i) AND F. LÉGARÉ ${ }^{1, *}$ \\ ${ }^{1}$ Institut National de la Recherche Scientifique (INRS), Centre EMT, ALLS Laboratory, 1650 Boulevard Lionel-Boulet, Varennes, Quebec J3X1S2, \\ Canada \\ ${ }^{2}$ Laboratoire d'Optique Appliquée, Ecole Polytechnique, ENSTA, CNRS, Université Paris Saclay, Palaiseau, France \\ ${ }^{3}$ Laboratoire Photonique Numérique et Nanosciences (LP2N), UMR 5298, CNRS-IOGS-Université Bordeaux, 33400 Talence, France \\ ${ }^{4}$ Institut Universitaire de France (IUF), 1 rue Descartes, 75231 Paris, France \\ ${ }^{*}$ Corresponding author: legare@emt.inrs.ca
}

Received 5 February 2020; revised 19 March 2020; accepted 20 March 2020; posted 23 March 2020 (Doc. ID 389804);

published 9 April 2020

\begin{abstract}
We present a novel, to the best of our knowledge, approach for scaling the peak power of mid-infrared laser pulses with few-cycle duration and carrier-to-envelope phase stabilization. Using frequency domain optical parametric amplification (FOPA), selective amplification is performed on two spectral slices of broadband pulses centered at $1.8 \mu \mathrm{m}$ wavelength. In addition to amplification, the Fourier plane is used for specific pulse shaping to control both the relative polarization and the phase/delay between the two spectral slices of the input pulses. At the output of the FOPA, intrapulse difference frequency generation provides carrier-envelope phase stabilized two-cycle pulses centered at $9.5 \mu \mathrm{m}$ wavelength with $25.5 \mu \mathrm{J}$ pulse energy. The control of the carrier-envelope phase is demonstrated through the dependence of high-harmonic generation in solids. This architecture is perfectly adapted to be scaled in the future to high average and high peak powers using picosecond ytterbium laser technologies. (๑) 2020 Optical Society of America
\end{abstract}

https://doi.org/10.1364/OL.389804

The attosecond revolution was possible through the development of laser technologies capable of generating high-energy few-cycle pulses at $800 \mathrm{~nm}$ (titanium-sapphire, Ti-Sa) with carrier-envelope phase (CEP) stability [1-3]. By driving highharmonic generation (HHG) with these laser fields, isolated attosecond pulses are generated and used for pump-probe experiments with the ultimate temporal resolution for real-time tracking of electronic dynamics in atoms $[1,4,5]$, molecules $[6,7]$, and solids [8,9]. However, for several applications, highfield pulses at longer wavelengths are required, thus motivating the development of sources from the infrared to the $\mathrm{THz}$ spectral range [10-16]. Among the applications, scaling the ponderomotive energy $\left(U_{p} \sim I \lambda^{2}\right)$ allows for the generation of attosecond pulses up to the soft $\mathrm{x}$-ray spectral range using the process of HHG in gas [17-19]. Moreover, it enables to perform photoelectron streaking with a combined large temporal window and a high temporal resolution [20], as well as driving HHG and sub-cycle electronic dynamics in low bandgap materials [21-24].

Independent of the central wavelength, most sources delivering long wavelength CEP stabilized pulses involve the process of difference frequency generation (DFG) between phase-locked frequencies, similar to passive CEP stabilization of the idler pulses obtained from white-light seeded optical parametric amplification (OPA) [25]. Here, we present a novel strategy to generate high-field CEP stabilized mid-infrared laser pulses based on frequency domain optical parametric amplification (FOPA) [26].

The FOPA is based on a $4 f$-setup where the input pulse spectra are spatially spread in the Fourier plane where broadband optical parametric amplification (OPA) is performed using several nonlinear crystals to optimize the phase matching and the gain of each spectral slice. This laser technology was previously demonstrated to amplify few-cycle IR pulses to a peak power of $2.5 \mathrm{TW}$ [27]. Here, rather than amplifying the full bandwidth of the IR pulses, selective amplification of two narrower spectral slices is performed in the Fourier plane. As illustrated in Fig. 1, this is done by splitting the high-energy pump into two beams, one for each spectral slice to be amplified. In addition to spectrally shaping the amplification, a half-wave plate is placed in the Fourier plane to rotate the polarization of one spectral slice to fulfill the phase-matching condition in the subsequent DFG stage. The optical delay introduced by the wave plate is compensated for by a window installed in the optical path of the second spectral slice (see Fig. 1). Following the FOPA, both amplified spectral slices are recombined and mixed in a type-II crystal of gallium selenide $(\mathrm{GaSe})$, generating high-field idler pulses that are passively CEP stabilized from the process of intrapulse DFG [25]. With this approach, $25.5 \mu \mathrm{J}$ energy, two-cycle pulses (66 fs 


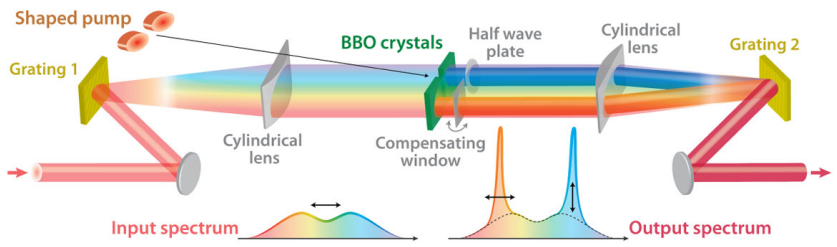

Fig. 1. Manipulation of the laser pulses in the Fourier plane of the FOPA prior to mid-IR generation by DFG. The schematic of the amplification of only two spectral slices is illustrated with the input and output spectra together with their polarizations at the bottom.

pulse duration), centered at a wavelength of $9.5 \mu \mathrm{m}$, are generated. The control of the CEP is demonstrated by measuring the dependence of the HHG spectra from solids as a function of the driving mid-IR field waveform.

The experiments were performed at the Advanced Laser Light Source facility located at INRS-EMT. The optical layout is illustrated in Fig. 2(a). The chirped pulse amplification (CPA) Ti-Sa laser system delivers femtosecond pulses at a repetition rate of $100 \mathrm{~Hz}$ with the central wavelength at $800 \mathrm{~nm}$. The output is divided into two synchronized beamlines with two independent compressors.

The first beamline delivers pulses with $4.5 \mathrm{~mJ}$ energy and $40 \mathrm{fs}$ duration and is used for pumping a commercial OPA (TOPAS, Light Conversion). At this OPA output, the idler pulses at the wavelength of $1.8 \mu \mathrm{m}$ are selected. Their pulse duration is $52 \mathrm{fs}$ with energy of $900 \mu \mathrm{J}$. Their spectra are presented in Fig. 2(b). As illustrated in panel (a), the beam is then coupled into a $2.7 \mathrm{~m}$ stretched hollow-core fiber filled with krypton at 1.7 bar of pressure [28], resulting in spectra broadened from 1.5 to $2.1 \mu \mathrm{m}$ [see panel (b)]. After the fiber, the broadband IR pulses then propagate through the FOPA composed of two gratings of 75 grooves $/ \mathrm{mm}$ and two cylindrical lenses of $+75 \mathrm{~cm}$. In the Fourier plane, the spectra are spatially spread over $\sim 3.4 \mathrm{~cm}$ in the horizontal direction (the dispersive direction of the gratings), and the beam diameter extends over $\sim 5.5 \mathrm{~mm}\left(1 / \mathrm{e}^{2}\right.$ diameter $)$ in the vertical direction. In this plane, the IR pulse duration is about 1 ps.

The second beamline of the laser system is used to pump the FOPA. By adjusting the grating distance in the compressor, its

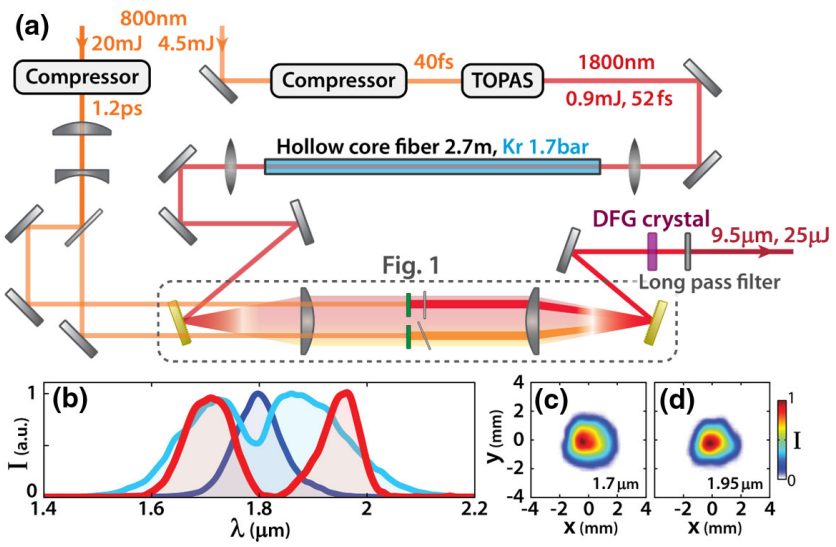

Fig. 2. (a) Schematic of the whole experimental setup. (b) Spectra of the IR beam at the entrance (dark blue) and output (light blue) of the stretch hollow-core fiber, and at the output of the FOPA (red). The spatial profiles of the amplified beam measured by two-photon absorption are shown for the (c) 1.7 and (d) $1.95 \mu \mathrm{m}$ spectral slices. pulse duration is set to $1.2 \mathrm{ps}$ [measured by second-harmonic generation frequency resolved optical gating (SHG-FROG [29])] to match the IR pulse duration in the Fourier plane. Following the compressor, the pump beam is shaped spatially in order to fit the spatial dimensions of both spectral slices to be amplified by using a telescope composed of two confocal cylindrical lenses $(+100 \mathrm{~cm}$ and $-50 \mathrm{~cm})$. The beam elliptical profiles obtained are $\sim 4 \mathrm{~mm}$ and $8 \mathrm{~mm}$ in the vertical and horizontal directions, respectively. Then, it is divided by a $50-50$ beam splitter into two separate beams for amplification of the two spectral slices.

As illustrated in Fig. 1, two $5 \mathrm{~mm}$ thick type-I beta-barium borate (BBO) crystals with dimensions of $5 \times 10 \times 20 \mathrm{~mm}^{3}$ are placed in the Fourier plane for parametric amplification. The polarizations of the pump and the seed are vertical and horizontal, respectively. The phase matching is adjusted by rotating each crystal around the horizontal axis perpendicular to the direction of propagation. To bring the pump beams in the amplifier, they propagate in a slightly non-collinear geometry: the angle between the IR and the pump beams is 2 deg in the vertical direction. The relative delay of each pump pulse is controlled independently by two delay lines, and the orientation of each BBO crystal is optimized for the amplification of each spectral slice. Right after the crystals in the Fourier plane, a half-wave plate is placed in the optical path of the 1.8 to $2.1 \mu \mathrm{m}$ part of the IR beam spectra to rotate its polarization by $90 \mathrm{deg}$. To compensate for the delay accumulated by propagation through the half-wave plate, a $1 \mathrm{~mm}$ thick fused silica window is positioned near the Brewster angle in the optical path of the second slice (spanning from 1.5 to $1.8 \mu \mathrm{m}$ ). This compensating window is mounted on a motorized rotation stage for precise control of the relative delay between both amplified slices.

The spectra of the IR beam at the output of the FOPA are shown in Fig. 2(b). With pump energies of 9.9 and $9.5 \mathrm{~mJ}$ $( \pm 2.1 \% \mathrm{rms}$ instability) for the amplification of the 1.7 and $1.95 \mu \mathrm{m}$ slices, energies of 1.21 and $1.27 \mathrm{~mJ}( \pm 2.25 \% \mathrm{rms})$ are obtained, respectively. The output beam profiles, measured at the FOPA output (after the second grating) using two-photon absorption in a silicon-based CCD camera (Flea2, PointGrey), are shown in panels (c) and (d) of Fig. 2. The pulse durations at 1.7 and $1.95 \mu \mathrm{m}$ are measured, respectively, to 55 and $100 \mathrm{fs}$ by SHG-FROG.

Generation of mid-infrared pulses with FOPA. After the FOPA, intrapulse DFG between the co-propagating spectral slices is performed by nonlinear conversion in a type-II GaSe crystal of $750 \mu \mathrm{m}$ thickness. A long-pass filter (LP6715, Spectrogon) selects only the spectral components above $6.7 \mu \mathrm{m}$. Mid-IR pulses of $25.5 \mu \mathrm{J}$ energy $( \pm 3.5 \% \mathrm{rms})$ centered at $9.5 \mu \mathrm{m}$ are obtained with spectra spanning from 7.2 to $13.5 \mu \mathrm{m}$, as presented in Fig. 3(d). The pulses are temporally characterized with the frequency resolved optical switching (FROSt) technique [30]. To perform this measurement, a few $\mu \mathrm{J}$ of energy of the mid-IR beam are focused with a $5 \mathrm{~mm}$ offaxis parabola onto a $300 \mu \mathrm{m}$ thick germanium $(\mathrm{Ge})$ plate. In parallel, the $1.95 \mu \mathrm{m}$ beam reflected by the long-pass filter (after the DFG) is frequency doubled to $\sim 1 \mu \mathrm{m}$ by a $2 \mathrm{~mm}$ thick type-I BBO crystal. These $\sim 120 \mu \mathrm{J}$ pulses are used to pump the Ge plate for the FROSt measurement. The transmitted mid-IR spectra through the Ge plate are measured as a function of the relative delay with the $1 \mu \mathrm{m}$ pulses by using a monochromator coupled to the $\mathrm{HgCdTe}$ (HCT) bolometric detector from 


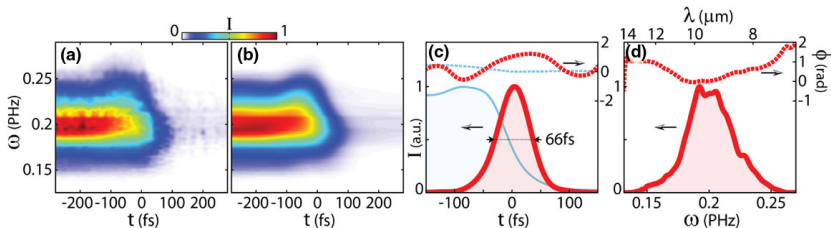

Fig. 3. FROSt characterization of the mid-IR pulses. Measured and reconstructed spectrograms in panels (a) and (b), respectively. Retrieved (c) temporal and (d) spectral profiles in intensity (solid red lines) and phase (dash red lines) of the mid-IR pulses. Temporal profile in intensity (solid blue line) and phase (dash blue line) of the optical switch are shown in panel (c). Note that both the frequency (bottom) and wavelength (top) scales are presented in panel (d).

Infrared Associates Inc. cooled down with liquid nitrogen. The measured and reconstructed FROSt spectrograms are presented in Figs. 3(a) and 3(b), respectively. In panel (c), the retrieved temporal intensity and phase profiles of the mid-IR pulses and the optical switch are shown. The spectral profiles of the mid-IR pulses are presented in panel (d) both in intensity and phase. The pulse duration is $66 \mathrm{fs}$ full width at half maximum (FWHM), as highlighted in Fig. 3(c), corresponding to nearly two optical cycles at $9.5 \mu \mathrm{m}$.

Note that from a simple calculation using the central wavelength of our two spectral slices, the generated mid-IR pulses should be at $13 \mu \mathrm{m}$ rather than $9.5 \mu \mathrm{m}$. While this is still under investigation, we refer to [15] where with a different laser system and DFG nonlinear crystal, they have observed $8.5 \mu \mathrm{m}$ rather than $10 \mu \mathrm{m}$. They explained their results by nonlinear propagation effects within the DFG crystal that translate into spectral reshaping of the incoming pulses. This will be further investigated for our approach as we are studying its spectral tunability.

CEP control using the FOPA. The approach presented here to generate mid-IR pulses is based on intrapulse DFG providing passive CEP stabilization [25]. With this setup, a slight rotation of the compensating window placed in the optical path of the $1.7 \mu \mathrm{m}$ slice induces a subtle change in its relative delay with the one at $1.95 \mu \mathrm{m}$ at the FOPA output. Thus, an accurate variation of this relative phase enables us to scan the CEP of the generated mid-IR pulses [31].

As previously shown [32-34], HHG in solids is highly sensitive to the CEP of the driving few-cycle pulses. This unique feature of HHG from solids allows us to confirm that the FOPA architecture enables not only the amplification of the two spectral slices, but also the control of their relative phases in order to tailor the CEP of the generated mid-IR pulses. For this, the $25.5 \mu \mathrm{J}$ mid-IR pulses are focused by the $50 \mathrm{~mm}$ off-axis parabola into a $1 \mathrm{~mm}$ thick window of zinc selenide ( $\mathrm{ZnSe}$ ) for HHG. At focus, the field strength is estimated to $\sim 45 \mathrm{MV} / \mathrm{cm}$. With two off-axis parabolic mirrors, the generated harmonics are collimated (confocal configuration) and re-focused into an IR spectrometer (NIR256-2.5, Ocean Optics) to measure the harmonic spectra from 0.8 to $2.5 \mu \mathrm{m}$. Figure 4 (a) shows the HHG spectra measured as a function of the CEP, controlled by rotating the compensating window. Each spectrum is obtained from the averaging of 200 shots $(2 \mathrm{~s}$ at $100 \mathrm{~Hz})$. The harmonic spectra exhibit a strong sensitivity to the CEP of the driving pulses with a shift of the harmonic peaks, as observed and explained previously [32,33]. In addition, panel (c) presents the

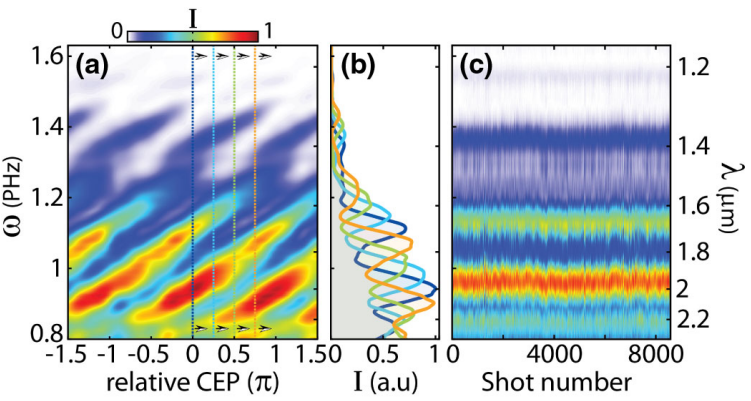

Fig. 4. (a) Harmonic spectra in intensity as a function of the relative CEP of the driving mid-IR pulse. (b) Four harmonic profiles for a relative CEP of zero (dark blue), $\pi / 4$ (light blue), $\pi / 2$ (green), and $3 \pi / 4$ (orange) are plotted. (c) Single-shot measurement of the harmonic spectrum for a fixed CEP of $\sim \pi / 10$ over more than 8000 shots. Note that both frequency (left) and wavelength (right) scales are presented.

harmonic spectrum measured in single-shot for a fixed relative CEP of $\sim \pi / 10$ over more than 8000 shots $(80 s$ at $100 \mathrm{~Hz})$. As can be observed, the spectrum remains very similar over time. These results make a clear demonstration that the CEP of the mid-IR pulses is passively stabilized and that the resulting waveforms can be tailored with our system.

In the mid-IR spectral range, it remains difficult and challenging to demonstrate the CEP control of ultrashort pulses, as the methods available in the near IR are not easily transferrable to long wavelengths. Therefore, when developing novel strategies for generating and amplifying CEP stabilized mid-IR pulses, many previously published studies are assuming that their source is CEP stabilized from the intrapulse DFG process without any experimental proof, and refer to the pioneering work of Baltuska and co-workers [25]. Here, we have used a simple and original method to demonstrate the CEP control by generating high harmonics in solids.

In conclusion, a novel strategy to generate high-field mid-IR CEP stabilized few-cycle pulses is introduced. Using a FOPA, the parametric amplification is shaped over two narrow spectral slices of IR broadband pulses, and the Fourier plane is used like a pulse shaper to manipulate the polarization and the phase of the amplified frequencies. Subsequently, the phase-locked amplified spectral slices are mixed in a nonlinear crystal for intrapulse DFG resulting in a passive stabilization of the CEP. Specifically, CEP stabilized two-cycle pulses centered at $9.5 \mu \mathrm{m}$ wavelength with $25.5 \mu \mathrm{J}$ energy are obtained.

Beyond the proof of concept presented in this Letter, this scheme is particularly promising to reach in the future highintensity mid-IR pulses. The input femtosecond IR pulses are temporally stretched in the Fourier plane of the 4f-setup up to picosecond duration; therefore, pulses with very high energy but low peak power compared to the femtosecond regime can be used to pump the OPA process. For instance, at $1.8 \mu \mathrm{m}$, this amplifier has enabled to reach $2.5 \mathrm{TW}$ of peak power using a pump of $200 \mathrm{~mJ}$ [27], promising to upscale our approach (currently $20 \mathrm{~mJ}$ of pump) by one order of magnitude in mid-IR pulse energy. On top of the high peak power, considering the use of a picosecond pump laser, the strategy presented in this Letter is perfectly adapted to ytterbium laser systems to obtain high average power [35-38]. 
Funding. H2020 Marie Skłodowska-Curie Actions (798205); Banting Research Foundation (201709BPF393834-285459); Institut Universitaire de France.

Disclosures. The authors declare no conflicts of interest.

\section{REFERENCES}

1. M. Hentschel, R. Kienberger, C. Spielmann, G. A. Reider, N. Milosevic, T. Brabec, P. Corkum, U. Heinzmann, M. Drescher, and F. Krausz, Nature 414, 509 (2001).

2. A. Baltuška, T. Udem, M. Uiberacker, M. Hentschel, E. Goulielmakis, C. Gohle, R. Holzwarth, V. Yakovlev, A. Scrinzi, T. W. Hänsch, and F. Krausz, Nature 421, 611 (2003).

3. G. Sansone, E. Benedetti, F. Calegari, C. Vozzi, L. Avaldi, R. Flammini, L. Poletto, P. Villoresi, C. Altucci, R. Velotta, S. Stagira, S. De Silvestri, and M. Nisoli, Science 314, 443 (2006).

4. E. Goulielmakis, Z.-H. Loh, A. Wirth, R. Santra, N. Rohringer, V. S. Yakovlev, S. Zherebtsov, T. Pfeifer, A. M. Azzeer, M. F. Kling, S. R. Leoni, and F. Krausz, Nature 466, 739 (2010).

5. M. Holler, F. Schapper, L. Gallmann, and U. Keller, Phys. Rev. Lett. 106, 123601 (2011).

6. G. Sansone, F. Kelkensberg, J. Pérez-Torres, F. Morales, M. F. Kling, W. Siu, O. Ghafur, P. Johnsson, M. Swoboda, E. Benedetti, F. Ferrari, F. Lépine, J.-L. Sanz-Vicario, S. Zherebtsov, I. Znakovskaya, A. L'Huillier, M. Y. Ivanov, M. Nisoli, F. Martin, and M. J. J. Vrakking, Nature 465, 763 (2010).

7. F. Calegari, D. Ayuso, A. Trabattoni, L. Belshaw, S. De Camillis, S. Anumula, F. Frassetto, L. Poletto, A. Palacios, P. Decleva, J. B. Greenwood, F. Martin, and M. Nisoli, Science 346, 336 (2014).

8. A. L. Cavalieri, N. Müller, T. Uphues, V. S. Yakovlev, A. Baltuška, B. Horvath, B. Schmidt, L. Blümel, R. Holzwarth, S. Hendel, M. Drescher, U. Kleineberg, P. M. Echenique, R. Kienberger, F. Krausz, and U. Heinzmann, Nature 449, 1029 (2007).

9. F. Siegrist, J. A. Gessner, M. Ossiander, C. Denker, Y.-P. Chang, M. C. Schröder, A. Guggenmos, Y. Cui, J. Walowski, U. Martens, J. K. Dewhurst, U. Kleineberg, M. Münzenberg, S. Sharma, and M. Schultze, Nature 571, 240 (2019).

10. C. Vozzi, F. Calegari, E. Benedetti, S. Gasilov, G. Sansone, G. Cerullo, M. Nisoli, S. De Silvestri, and S. Stagira, Opt. Lett. 32, 2957 (2007).

11. B. E. Schmidt, A. D. Shiner, P. Lassonde, J.-C. Kieffer, P. B. Corkum, D. M. Villeneuve, and F. Légaré, Opt. Express 19, 6858 (2011).

12. D. Sanchez, M. Hemmer, M. Baudisch, S. Cousin, K. Zawilski, P. Schunemann, O. Chalus, C. Simon-Boisson, and J. Biegert, Optica 3, 147 (2016)

13. A. Sell, A. Leitenstorfer, and R. Huber, Opt. Lett. 33, 2767 (2008).

14. P. Krogen, H. Suchowski, H. Liang, N. Flemens, K.-H. Hong, F. X. Kärtner, and J. Moses, Nat. Photonics 11, 222 (2017).

15. O. Novák, P. R. Krogen, T. Kroh, T. Mocek, F. X. Kärtner, and K.-H. Hong, Opt. Lett. 43, 1335 (2018).

16. K. Liu, H. Liang, L. Wang, S. Qu, T. Lang, H. Li, Q. J. Wang, and Y. Zhang, Opt. Lett. 44, 1003 (2019).
17. N. Ishii, K. Kaneshima, K. Kitano, T. Kanai, S. Watanabe, and J. Itatani, Nat. Commun. 5, 3331 (2014).

18. S. M. Teichmann, F. Silva, S. Cousin, M. Hemmer, and J. Biegert, Nat. Commun. 7, 11493 (2016).

19. J. Li, X. Ren, Y. Yin, K. Zhao, A. Chew, Y. Cheng, E. Cunningham, Y. Wang, S. Hu, Y. Wu, M. Chini, and Z. Chang, Nat. Commun. 8, 186 (2017).

20. U. Frühling, M. Wieland, M. Gensch, T. Gebert, B. Schütte, M. Krikunova, R. Kalms, F. Budzyn, O. Grimm, J. Rossbach, E. Plönjes, and M. Drescher, Nat. Photonics 3, 523 (2009).

21. S. Ghimire, A. D. DiChiara, E. Sistrunk, P. Agostini, L. F. DiMauro, and D. A. Reis, Nat. Phys. 7, 138 (2011).

22. G. Vampa, T. Hammond, N. Thiré, B. Schmidt, F. Légaré, C. McDonald, T. Brabec, and P. Corkum, Nature 522, 462 (2015).

23. M. Hohenleutner, F. Langer, O. Schubert, M. Knorr, U. Huttner, S. W. Koch, M. Kira, and R. Huber, Nature 523, 572 (2015).

24. J. Reimann, S. Schlauderer, C. Schmid, F. Langer, S. Baierl, K. Kokh, O. Tereshchenko, A. Kimura, C. Lange, J. Güdde, U. Höfer, and R. Huber, Nature 562, 396 (2018).

25. A. Baltuška, T. Fuji, and T. Kobayashi, Phys. Rev. Lett. 88, 133901 (2002).

26. B. E. Schmidt, N. Thiré, M. Boivin, A. Laramée, F. Poitras, G. Lebrun, T. Ozaki, H. Ibrahim, and F. Légaré, Nat. Commun. 5, 3643 (2014).

27. V. Gruson, G. Ernotte, P. Lassonde, A. Laramée, M. Bionta, M. Chaker, L. Di Mauro, P. Corkum, H. Ibrahim, B. Schmidt, and F. Légaré, Opt. Express 25, 27706 (2017).

28. V. Cardin, N. Thiré, S. Beaulieu, V. Wanie, F. Légaré, and B. E. Schmidt, Appl. Phys. Lett. 107, 181101 (2015).

29. R. Trebino, Frequency-Resolved Optical Gating: The Measurement of Ultrashort Laser Pulses (Springer, 2012).

30. A. Leblanc, P. Lassonde, S. Petit, J.-C. Delagnes, E. Haddad, G. Ernotte, M. Bionta, V. Gruson, B. Schmidt, H. Ibrahim, E. Cormier, and F. Légaré, Opt. Express 27, 28998 (2019).

31. G. Ernotte, P. Lassonde, F. Légaré, and B. Schmidt, Opt. Express 24 , 24225 (2016)

32. Y. S. You, M. Wu, Y. Yin, A. Chew, X. Ren, S. Gholam-Mirzaei, D. A Browne, M. Chini, Z. Chang, K. J. Schafer, J. Schafer, M. B. Gaarde, and S. Ghimire, Opt. Lett. 42, 1816 (2017).

33. Y. S. You, Y. Yin, Y. Wu, A. Chew, X. Ren, F. Zhuang, S. GholamMirzaei, M. Chini, Z. Chang, and S. Ghimire, Nat. Commun. 8, 724 (2017).

34. X. Song, R. Zuo, S. Yang, P. Li, T. Meier, and W. Yang, Opt. Express 27, 2225 (2019).

35. C. Baumgarten, M. Pedicone, H. Bravo, H. Wang, L. Yin, C. S. Menoni, J. J. Rocca, and B. A. Reagan, Opt. Lett. 41, 3339 (2016).

36. H. Fattahi, A. Alismail, H. Wang, J. Brons, O. Pronin, T. Buberl, L. Vámos, G. Arisholm, A. M. Azzeer, and F. Krausz, Opt. Lett. 41, 1126 (2016).

37. B. E. Schmidt, A. Hage, T. Mans, F. Légaré, and H. J. Wörner, Opt Express 25, 17549 (2017).

38. M. Kienel, M. Müller, A. Klenke, J. Limpert, and A. Tünnermann, Opt. Lett. 41, 3343 (2016) 